\title{
Convexity properties of coverings of 1-convex surfaces *
}

\author{
M. Colţoiu and C. Joiţa
}

\begin{abstract}
We prove that there exists a 1-convex surface whose universal covering does not satisfy the discrete disk property.
\end{abstract}

\section{Introduction}

The well-known Shafarevich Conjecture asserts that the universal covering space of a projective algebraic manifold is holomorphically convex. Although there are partial results, a complete answer to this problem is not known even for surfaces. (We remark that if instead of the universal covering one considers an arbitrary non-compact one, there are counterexamples, see [7]).

In this paper we are interested in studying convexity properties of the universal covering of 1-convex surfaces. We recall that projective algebraic manifolds are a particular case of Moishezon manifolds, that the exceptional set of a 1-convex manifold is a Moishezon space and that every Moishezon space is the exceptional set of a 1-convex manifold.

Suppose that $X$ is a 1 -convex surface and $p: \tilde{X} \rightarrow X$ is a covering map. It is known (see [1]) that in general $\tilde{X}$ is not holomorphically convex. In fact $\tilde{X}$ might not be even weakly 1-complete (that is, $\tilde{X}$ might not carry a continuous plurisubharmonic exhaustion function). However $\tilde{X}$ can be exhausted by a sequence of strongly pseudoconvex domains and therefore $\tilde{X}$ satisfies the continuous disk property (see the next section for a precise definition). We investigate the discrete disk property for $\tilde{X}$ which definitely is a stronger property.

*Mathematics Subject Classification (2000): 32E05, 32F10.

Key words: holomorphically convex manifolds, 1-convex manifolds, universal covering. 
Our main goal is to give an example of a 1-convex surface whose universal covering does not satisfy the discrete disk property. In particular it will not be $p_{5}$-convex in the sense of [3]. We proved in [2] that if $\tilde{X}$ does not contain an infinite Nori string of rational curves then actually $\tilde{X}$ does satisfy the discrete disk property. Therefore our example must contain such a Nori string.

We remark that important convexity properties of coverings of 1-convex manifolds with respect to meromorphic functions have been established in [7.

\section{Preliminaries}

We denote by $\Delta$ the unit disk in $\mathbb{C}, \Delta=\{z \in \mathbb{C}:|z|<1\}$ and for $c>0$ by $\Delta_{1+c}$ the disk $\Delta_{1+c}:=\{z \in \mathbb{C}:|z|<1+c\}$.

For $\epsilon>0$ we define $H_{\epsilon} \subset \mathbb{C} \times \mathbb{R}$ as

$$
H_{\epsilon}=\Delta_{1+\epsilon} \times[0,1) \bigcup\{z \in \mathbb{C}: 1-\epsilon<|z|<1+\epsilon\} \times\{1\} .
$$

The following is just an intrinsic version of the classical Continuity Principle (see, for example [6] page 47).

Definition 1. A complex space $X$ is said to satisfy the continuous disk property if whenever $\epsilon$ is a positive number and $F: H_{\epsilon} \rightarrow X$ is a continuous function such that, for every $t \in[0,1), F_{t}: \Delta_{1+\epsilon} \rightarrow X, F_{t}(z)=F(z, t)$, is holomorphic we have that $F\left(H_{\epsilon_{1}}\right)$ is relatively compact in $X$ for any $0<\epsilon_{1}<\epsilon$.

Motivated by the above definition we introduced in [2]:

Definition 2. Suppose that $X$ is a complex space. We say that $X$ satisfies the discrete disk property if whenever $g_{n}: U \rightarrow X$ is a sequence of holomorphic functions defined on an open neighborhood $U$ of $\bar{\Delta}$ for which there exists an $\epsilon>0$ and a continuous function $\gamma: S^{1}=\{z \in \mathbb{C}:|z|=1\} \rightarrow X$ such that $\Delta_{1+c} \subset U, \bigcup_{n \geq 1} g_{n}\left(\Delta_{1+\epsilon} \backslash \Delta\right)$ is relatively compact in $X$ and $g_{n \mid S^{1}}$ converges uniformly to $\gamma$ we have that $\bigcup_{n \geq 1} g_{n}(\bar{\Delta})$ is relatively compact in $X$.

Note that if a complex space is $p_{5}$-convex in the sense of Docquier and Grauert [3] then it satisfies the discrete disk property. Therefore our example will not be $p_{5}$-convex either. $X$ is called $p_{5}$-convex if whenever $\left\{\Delta_{\nu}\right\}_{\nu \geq 0}$ is a sequence of holomorphic disks such that $\bigcup_{\nu \geq 0} \partial \Delta_{\nu} \Subset X$ we have that $\bigcup_{\nu \geq 0} \bar{\Delta}_{\nu} \Subset X$ as well. 
In [4] it is constructed a complex manifold which is an increasing union of Stein open subsets, and therefore it satisfies the continous disk property, but it does not satisfy the discrete disk property. In particular this shows that the discrete disk property is stronger that the continuous one.

We recall that a compact complex curve is called rational if its normalization is $\mathbb{P}^{1}$.

A complex manifold is called 1-convex if it is the modification of a Stein space at a finite set of points.

Definition 3. Let $L$ be a connected 1-dimensional complex space and $\cup L_{i}$ be its decomposition into irreducible components. L is called an infinite Nori string if all $L_{i}$ are compact and $L$ is not compact

The following theorem was proved in [2].

Theorem 1. Let $X$ be a 1-convex surface and $p: \tilde{X} \rightarrow X$ be a covering map. If $\tilde{X}$ does not contain an infinite Nori string of rational curves then $\tilde{X}$ satisfies the discrete disk property.

\section{The Results}

As we mentioned in the introduction, our goal is to prove the following theorem.

Theorem 2. There exists a 1-convex surface whose universal covering does not satisfy the discrete disk property.

We will describe first the basic idea of the proof of the theorem. We start with a basic example of a 2-dimensional complex manifold $X$ that does not satisfy the discrete disk property and contains an infinite Nori string of rational curves. Let $f_{n}: \mathbb{C} \rightarrow \mathbb{C}^{2}, f(\lambda)=\left(\left(\frac{\lambda}{2}\right)^{n}, \lambda\right)$. We consider the complex manifold which is obtained from $\mathbb{C}^{2}$ after an infinite sequence of blow-ups. The center of a blow-up is chosen to be a point on the exceptional divisor of the previous blow-up. More precisely, for $k \leq n-1$ the center of the $k^{t h}$ blow-up is the image of the origin through the the proper transform of $f_{n}$. It is not difficult to see that the manifold thus obtained does not satisfy the discrete disk property. It contains a Nori string $\left\{L_{k}\right\}_{k \geq 0}$ of curves isomorphic to $\mathbb{P}^{1}$. Going backwards we can construct from $X$ a manifold $Y$ that contains a Nori string $\left\{L_{k}\right\}_{k \in \mathbb{Z}}$ (so $L_{k}$ is defined also for $k<0$ ). Then $\bigcup_{k \in \mathbb{Z}} L_{k}$ will 
cover $F_{0} \cup F_{1}$ where $F_{0}$ and $F_{1}$ are isomorphic to $\mathbb{P}^{1}$ and $F_{0} \cap F_{1}$ has exactly two points. An appropriately chosen neighborhood $U$ of $\bigcup_{k \in \mathbb{Z}} L_{k}$ in $Y$ will cover a manifold $V$ which is a neighborhood of $F_{0} \cup F_{1}$. It is again not very hard to prove that $U$ does not satisfy the discrete disk property. However $F_{0} \cup F_{1}$ is not exceptional because the intersection matrix is

$$
\left[\begin{array}{cc}
-2 & 2 \\
2 & -2
\end{array}\right]
$$

and then we have to blow-up again at two points, one on $F_{0}$ and one on $F_{1}$ (hence we have to blow-up $L_{k}$ accordingly) in order to make the intersection matrix negative defined. To show that the manifold $Z$ obtained in this way does not satisfy the discrete disk property is not easy anymore. A sequence of holomorphic disks defined in the simple-minded way as the one above will not work because their image will not stay in a small enough neighborhood in $Z$ of the proper transform of $\bigcup_{k \in \mathbb{Z}} L_{k}$.

We move now to the proof of our theorem.

Step 1. We construct a 1-convex manifold $W$ and a covering $\tilde{p}: \tilde{W} \rightarrow W$. In the second step we will show that $\tilde{W}$ does not have the discrete disk property.

Suppose that $M$ is a two-dimensional complex manifold, $a \in M$ is a point and $\left(z_{1}, z_{2}\right): U \rightarrow \mathbb{C}^{2}$ is a local chart around $a$ such that $\left(z_{1}(a), z_{2}(a)\right)=$ $(0,0)$. We will define a complex manifold $\mathcal{I}\left(M,\left(z_{1}, z_{2}\right)\right)$ as follows.

We assume first that $M=\Omega_{0}=\mathbb{C}^{2},\left(z_{1}, z_{2}\right)=\left(z_{1}^{(0)}, z_{2}^{(0)}\right)$ are the coordinate functions and $a=a_{0}=(0,0)$. We define $\Omega_{k}$ to be the manifold obtained from $\Omega_{0}$ by performing a sequence of $k$ successive blow-ups as follows. Let $\Omega_{1}$ be the blow-up of $\Omega_{0}$ in $a_{0}$. Namely $\Omega_{1}=\left\{\left(z_{1}^{(0)}, z_{2}^{(0)},\left[\xi_{1}^{(0)}: \xi_{2}^{(0)}\right]\right) \in \Omega_{0} \times \mathbb{P}^{1}\right.$ : $\left.z_{1}^{(0)} \xi_{2}^{(0)}=z_{2}^{(0)} \xi_{1}^{(0)}\right\}$. Let $a_{1}=(0,0,[0: 1]) \in \Omega_{1}$ and $\Omega_{2}$ be the blow up of $\Omega_{1}$ in $a_{1}$ and let $L_{0}$ be the proper transform of the exceptional set of $\Omega_{1}$. The open subset of $\Omega_{1}$ given by $\xi_{2}^{(0)} \neq 0$ is biholomorphic to $\mathbb{C}^{2}$ with the coordinate functions $z_{1}^{(1)}:=\frac{\xi_{1}^{(0)}}{\xi_{2}^{(0)}}$ and $z_{2}^{(1)}:=z_{2}^{(0)}$. In these coordinates $a_{1}$ is given by $z_{1}^{(1)}=0, z_{2}^{(1)}=0$. We continue this procedure $k$ times and we obtain $\Omega_{k}$. In doing so we obtain also $L_{0}, \ldots L_{k-1}$, which are complex curves each one of them isomorphic to $\mathbb{P}^{1}$, and $a_{0}, a_{1}, \ldots, a_{k}$ the points where we are blowing up. Note that $\Omega_{j} \backslash\left\{a_{j}\right\}$ is an open subset of $\Omega_{j+1} \backslash\left\{a_{j+1}\right\}$. We set

$$
\mathcal{I}\left(\Omega_{0},\left(z_{1}^{(0)}, z_{2}^{(0)}\right)\right):=\cup_{j=0}^{\infty} \Omega_{j} \backslash\left\{a_{j}\right\}
$$


Notice that we have also a canonical map $\pi: \mathcal{I}\left(\Omega_{0},\left(z_{1}^{(0)}, z_{2}^{(0)}\right)\right) \rightarrow \mathbb{C}^{2}$ such that $\pi^{-1}(0)=\bigcup_{k \geq 0} L_{k}$ and $\pi: \mathcal{I}\left(\Omega_{0},\left(z_{1}^{(0)}, z_{2}^{(0)}\right)\right) \backslash \bigcup_{k \geq 0} L_{k} \rightarrow \mathbb{C}^{2} \backslash\{0\}$ is a biholomorphism. If $U$ is an open subset of $\mathbb{C}^{2}$ containing the origin we set $\mathcal{I}\left(U,\left(z_{1}, z_{2}\right)\right):=\pi^{-1}(U)$. Finally $\mathcal{I}\left(M,\left(z_{1}, z_{2}\right)\right)$ is defined by gluing $M \backslash\{a\}$ and $\mathcal{I}\left(U,\left(z_{1}, z_{2}\right)\right)$ via the identification of $U \backslash\{a\}$ with $\mathcal{I}\left(U,\left(z_{1}, z_{2}\right)\right) \backslash \bigcup_{k \geq 0} L_{k}$.

Notice that if $M$ is a complex manifold $\Omega$ is an open subset of $M, a$ is a point of $\Omega$ and $\left(z_{1}, z_{2}\right): U \rightarrow \mathbb{C}^{2}$ is a local chart around $a$, with $U \subset \Omega$ and $\left(z_{1}(a), z_{2}(a)\right)=(0,0)$, then $\mathcal{I}\left(\Omega,\left(z_{1}, z_{2}\right)\right)$ is an open subset of $\mathcal{I}\left(M,\left(z_{1}, z_{2}\right)\right)$

Next we will define inductively a sequence $\left\{X_{k}\right\}_{k \leq 0}$ of complex manifolds as follows. We consider $\mathbb{C}^{2}$ with coordinate functions $\left(z_{1}^{(0)}, z_{2}^{(0)}\right)$ and we set $X_{0}=\mathcal{I}\left(\mathbb{C}^{2},\left(z_{1}^{(0)}, z_{2}^{(0)}\right)\right)$. To define $X_{-1}$ we let $M_{-1}$ be the blow-up of $\mathbb{C}^{2}$ at the origin, written in coordinates as follows: $M_{-1}=\left\{\left(z_{1}^{(-1)}, z_{2}^{(-1)},\left[\xi_{1}^{(-1)}\right.\right.\right.$ : $\left.\left.\left.\xi_{2}^{(-1)}\right]\right) \in \mathbb{C}^{2} \times \mathbb{P}^{1}: z_{1}^{(-1)} \xi_{2}^{(-1)}=z_{2}^{(-1)} \xi_{1}^{(-1)}\right\}$. Then $\Omega_{-1}=\left\{\left(z_{1}^{(-1)}, z_{2}^{(-1)},\left[\xi_{1}^{(-1)}\right.\right.\right.$ : $\left.\left.\left.\xi_{2}^{(-1)}\right]\right) \in M_{-1}: \xi_{2}^{(-1)} \neq 0\right\}$ is an open set of $M_{-1}$, biholomorphic to $\mathbb{C}^{2}$ with coordinate functions $z_{1}^{(0)}:=\frac{\xi_{1}^{(-1)}}{\xi_{2}^{(-1)}}$ and $z_{2}^{(0)}:=z_{2}^{(-1)}$. We set $X_{-1}=$ $\mathcal{I}\left(M_{-1},\left(z_{1}^{(0)}, z_{2}^{(0)}\right)\right)$. Notice then that $X_{0}$ is an open subset of $X_{-1}$ and that $X_{-1}$ is biholomorphic to $X_{0}$. In the same way we defined $X_{-1}$ starting with $X_{0}$ we define $X_{k-1}$ starting with $X_{k}$.

We put $X=\bigcup_{k=0}^{-\infty} X_{k}$ and $L=\bigcup_{k=-\infty}^{\infty} L_{k}$. Notice that if $|j-k| \geq 2$ then $L_{j} \cap L_{k}=\emptyset$.

Next we want to define a fundamental system of open neighborhoods of $L_{k}$ for each $k \in \mathbb{Z}$. To do that we notice that, by construction, $L_{k}$ is obtained as follows: we have $\mathbb{C}^{2}$ with coordinate functions $\left(z_{1}^{(k)}, z_{2}^{(k)}\right)$ we blow it up at the origin and then we blow it up again at the point $(0,0,[0: 1])$. The manifold thus obtained is denoted by $\widehat{\mathbb{C}}^{2}$. Then $L_{k}$ is the proper transform of the exceptional set of the first blow-up. That is we have that $\widehat{\mathbb{C}}^{2}$ is given in $\mathbb{C}^{2} \times \mathbb{P}^{1} \times \mathbb{P}^{1}$ with coordinates $\left(z_{1}^{(k)}, z_{2}^{(k)},\left[\xi_{1}^{(k)}: \xi_{2}^{(k)}\right],\left[\xi_{1}^{(k+1)}: \xi_{2}^{(k+1)}\right]\right)$ by

$$
z_{1}^{(k)} \xi_{2}^{(k)}=z_{2}^{(k)} \xi_{1}^{(k)}, \xi_{1}^{(k)} \xi_{2}^{(k+1)}=\xi_{1}^{(k+1)} \xi_{2}^{(k)} z_{2}^{(k)}
$$

In $\widehat{\mathbb{C}}^{2}, L_{k}$ is given by the equations $z_{1}^{(k)}=0, \xi_{2}^{(k+1)}=0$.

For $r \in(0,1]$ we define $U_{r}^{(k)}:=\left\{\left|\xi_{2}^{(k+1)}\right|<r\left|\xi_{1}^{(k+1)}\right|,\left|z_{1}^{(k)}\right|<r\right\}$ and we notice that $\left\{U_{r}^{(k)}\right\}_{r>0}$ is indeed a fundamental system of open neighborhoods of $L_{k}$. Obviously $U_{r}^{(j)}$ and $U_{r}^{(k)}$ are biholomorphic for every $j$ and $k$.

We want to show that if $|j-k| \geq 2$ then $U_{r}^{(j)} \cap U_{r}^{(k)}=\emptyset$. It is clear from our construction that without loss of generality we can assume that 
$j=0$ and $k \geq 2$. As $U_{r}^{(j)} \cap U_{r}^{(k)}$ is an open set, it suffices to show that $\left(U_{r}^{(0)} \backslash L\right) \cap\left(U_{r}^{(k)} \backslash L\right)=\emptyset$. We recall that we have defined $z_{1}^{(k+1)}=\frac{\xi_{1}^{(k)}}{\xi_{2}^{(k)}}$ and $z_{2}^{(k+1)}=z_{2}^{(k)}$. Hence, outside $L$ and for $k \geq 0$, we have that $\left[z_{1}^{(k+1)}: z_{2}^{(k+1)}\right]=$ $\left[\xi_{1}^{(k)}: \xi_{2}^{(k)} z_{2}^{(k)}\right]=\left[z_{1}^{(k)}: z_{2}^{(k)} z_{2}^{(0)}\right]$. Inductively we get $\left[z_{1}^{(k+1)}: z_{2}^{(k+1)}\right]=\left[z_{1}^{(0)}:\right.$ $\left(z_{2}^{(0)}\right)^{k+2}$. The inequality $\left|z_{1}^{(k)}\right|<r$ is equivalent to $\left|\xi_{1}^{(k-1)}\right|<r\left|\xi_{2}^{(k-1)}\right|$. As $\left[\xi_{1}^{(j)}: \xi_{2}^{(j)}\right]=\left[z_{1}^{(j)}: z_{2}^{(j)}\right]$ for every $j \in \mathbb{Z}$ and every point in $X \backslash L$ it follows that

$$
U_{r}^{(k)} \backslash L=\left\{\left(z_{1}^{(0)}, z_{2}^{(0)}\right) \in \mathbb{C}^{2}:\left|z_{2}^{(0)}\right|^{k+2}<r\left|z_{1}^{(0)}\right|,\left|z_{1}^{(0)}\right|<r\left|z_{2}^{(0)}\right|^{k}\right\} .
$$

We have that $U_{r}^{(0)} \backslash L=\left\{\left(z_{1}^{(0)}, z_{2}^{(0)}\right) \in \mathbb{C}^{2}:\left|z_{2}^{(0)}\right|^{2}<r\left|z_{1}^{(0)}\right|,\left|z_{1}^{(0)}\right|<r\right\}$. In particular every point of $U_{r}^{(0)} \backslash L$ satisfies $\left|z_{2}^{(0)}\right|^{2}<r\left|z_{1}^{(0)}\right|<r^{2}$, hence $\left|z_{2}^{(0)}\right|<r$. Then a point in the intersection $\left(U_{r}^{(0)} \backslash L\right) \cap\left(U_{r}^{(k)} \backslash L\right)$ would satisfy $\left|z_{2}^{(0)}\right|^{2}<r\left|z_{1}^{(0)}\right|<r^{2}\left|z_{2}^{(0)}\right|^{k}$. As $k \geq 2$ we get $1<r^{2}\left|z_{2}^{(0)}\right|^{k-2}<r^{k}$ and this contradicts our choice of $r \leq 1$.

It is clear that the mapping $\left(z_{1}^{(k)}, z_{2}^{(k)},\left[\xi_{1}^{(k)}: \xi_{2}^{(k)}\right],\left[\xi_{1}^{(k+1)}: \xi_{2}^{(k+1)}\right]\right) \rightarrow$ $\left(z_{1}^{(j)}, z_{2}^{(j)},\left[\xi_{1}^{(j)}: \xi_{2}^{(j)}\right],\left[\xi_{1}^{(j+1)}: \xi_{2}^{(j+1)}\right]\right)$ induces a biholomorphism of $p_{k, j}$ : $U_{r}^{(k)} \rightarrow U_{r}^{(j)}$. Let $Y:=U_{1}^{(0)} \cup U_{1}^{(1)} / \sim$, where $\sim$ identifies $U_{1}^{(2)} \cup U_{1}^{(1)}$ to $U_{1}^{(-1)} \cup U_{1}^{(0)}$ via $p_{2,0}$. Let $\mathcal{U}=\bigcup_{k \in \mathbb{Z}} U_{1}^{(k)}$ and $p: \mathcal{U} \rightarrow Y$ be the map given by $p_{\mid U_{1}^{(2 k)}}=p_{2 k, 0}$ and $p_{\mid U_{1}^{(2 k+1)}}=p_{2 k+1,1}$. Clearly $p$ is a covering map. $F_{0}:=p\left(L_{0}\right)$ and $F_{1}:=p\left(L_{1}\right)$ are both biholomorphic to $\mathbb{P}^{1}$ and, moreover, we have $F_{0} \cdot F_{0}=-2, F_{1} \cdot F_{1}=-2, F_{0} \cdot F_{1}=2$. Let $\alpha_{k} \in L_{k}$ be the point given by $\left(z_{1}^{(k)}, z_{2}^{(k)},\left[\xi_{1}^{(k)}: \xi_{2}^{(k)}\right],\left[\xi_{1}^{(k+1)}: \xi_{2}^{(k+1)}\right]\right)=(0,0,[1: 1],[1: 0])$ and $\beta_{0}, \beta_{1} \in Y$ the points $\beta_{0}=p\left(\alpha_{2 k}\right), \beta_{1}=p\left(\alpha_{2 k+1}\right)$. We let $\pi: \tilde{Y} \rightarrow Y$ to be the blow up of $Y$ at $\beta_{0}$ and $\beta_{1}$ and we denote by $\tilde{F}_{0}$ and $\tilde{F}_{1}$ respectively the proper transforms of $F_{0}$ and $F_{1}$. Note that $\tilde{F}_{0} \cdot \tilde{F}_{0}=-3, \tilde{F}_{1} \cdot \tilde{F}_{1}=-3, \tilde{F}_{0} \cdot \tilde{F}_{1}=2$. As the intersection matrix

$$
\left[\begin{array}{cc}
-3 & 2 \\
2 & -3
\end{array}\right]
$$

is negative definite, it follows, see [5], that $\tilde{F}:=\tilde{F}_{0} \cup \tilde{F}_{1}$ is exceptional. We consider the following diagram:

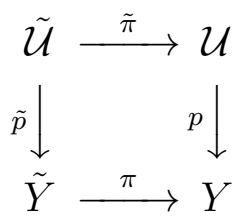


We let $\tilde{p}: \tilde{\mathcal{U}} \rightarrow \tilde{Y}$ be the pull-back of $p$. Clearly $\tilde{p}$ is a covering map and $\tilde{\pi}: \tilde{\mathcal{U}} \rightarrow \mathcal{U}$ is obtained by blowing-up $\mathcal{U}$ at every $\alpha_{k}, k \in \mathbb{Z}$. We choose now $W$ a 1-convex neighborhood of $\tilde{F}$ and we put $\tilde{W}:=\tilde{p}^{-1}(W), \tilde{L}:=\tilde{p}^{-1}(\tilde{F})$. If $\tilde{L}_{k}$ is the proper transform of $L_{k}$ in $\tilde{\mathcal{U}}$ then $\tilde{L}=\bigcup \tilde{L}_{k}$. We will show that $\tilde{W}$ does not have the discrete disk property. In our construction of the sequence of holomorphic discs we want to make sure that their image stays in $\tilde{W}$. To do that we need a "concrete" open neighborhood of $\tilde{L}$ in $\tilde{W}$. To obtain it we consider $\left\{\tilde{W}_{r, \rho}^{(k)}\right\}$ a fundamental system of neighborhoods for $\tilde{L}_{k}$, each one of them being actually the preimage via $\tilde{\pi}$ of a cone centered at $\alpha_{k}$. Moreover $p_{k, j}$ induces a biholomorphism $\tilde{W}_{r, \rho}^{(k)} \rightarrow \tilde{W}_{r, \rho}^{(j)}$. The construction is as follows.

We have the following description of the blow-up of $U_{1}^{(k)}$ in $\alpha_{k}$ : it is the set $\tilde{U}_{1}^{(k)}$ of all

$$
\left(z_{1}^{(k)}, z_{2}^{(k)},\left[\xi_{1}^{(k)}: \xi_{2}^{(k)}\right],\left[\xi_{1}^{(k+1)}: \xi_{2}^{(k+1)}\right],\left[w_{1}: w_{2}\right]\right) \in \mathbb{C}^{2} \times \mathbb{P}^{1} \times \mathbb{P}^{1} \times \mathbb{P}^{1}
$$

such that

$$
z_{1}^{(k)} \xi_{2}^{(k)}=z_{2}^{(k)} \xi_{1}^{(k)}, \xi_{1}^{(k)} \xi_{2}^{(k+1)}=\xi_{1}^{(k+1)} \xi_{2}^{(k)} z_{2}^{(k)}, w_{2} z_{1}^{(k)} \xi_{1}^{(k)}=w_{1}\left(\xi_{1}^{(k)}-\xi_{2}^{(k)}\right)
$$

and

$$
\left|z_{1}^{(k)}\right|<1,\left|\xi_{2}^{(k+1)}\right|<\left|\xi_{1}^{(k+1)}\right|
$$

The proper transform of $L_{k}$ is given by $z_{1}^{(k)}=0, \xi_{2}^{(k+1)}=0, w_{1}=0$. A fundamental system of neighborhoods for $\tilde{L}_{k}$ is given by

$$
\tilde{W}_{r, \rho}^{(k)}=\left\{\left|z_{1}^{(k)}\right|<r,\left|\xi_{2}^{(k+1)}\right|<r\left|\xi_{1}^{(k+1)}\right|,\left|w_{1}\right|<\rho\left|w_{2}\right|\right\} \subset \tilde{U}_{1}^{(k)} .
$$

There exist then $\rho>0$ and $r>0$ such that $\tilde{W}_{r}^{\rho}=\bigcup_{k \in \mathbb{Z}} \tilde{W}_{r, \rho}^{(k)} \subset \tilde{W}$.

If we denote by $W_{r}^{\rho} \subset \mathcal{U}$ the set

$$
\bigcup_{k \in \mathbb{Z}}\left\{\left(z_{1}^{(k)}, z_{2}^{(k)},\left[\xi_{1}^{(k)}: \xi_{2}^{(k)}\right],\left[\xi_{1}^{(k+1)}: \xi_{2}^{(k+1)}\right]\right) \in U_{r}^{(k)}:\left|z_{1}^{(k)} \xi_{1}^{(k)}\right|<\rho\left|\xi_{2}^{(k)}-\xi_{1}^{(k)}\right|\right\}
$$

we have that $\tilde{W} \backslash \tilde{L} \supset \tilde{W}_{r}^{\rho} \backslash \tilde{L} \supset W_{r}^{\rho} \backslash L$. We notice at the same time that keeping $\rho \in(0,1)$ fixed and choosing a small enough $r>0$ we have that $\tilde{W}_{r, \rho}^{(k)} \cap \tilde{W}_{r, \rho}^{(k+1)}=U_{r}^{(k)} \cap U_{r}^{(k+1)}$ for every $k \in \mathbb{Z}$. We fix such an $r \in(0,1)$ that satisfies also $r \leq \frac{\rho}{2}(1-r)$.

Step 2. We construct a sequence of holomorphic discs that proves that $\tilde{W}$ does not have the discrete disk property. 
We fix $n \in \mathbb{N}$. To define our $n^{\text {th }}$ holomorphic disk, $g_{n}$, we will start with two polynomial functions $f_{1}=f_{1}^{(n)}$ and $f_{2}=f_{2}^{(n)}$ and $g_{n}$ will be the proper transform of $\left(f_{1}, f_{2}\right): \mathbb{C} \rightarrow \Omega_{0}$ restricted to a neighborhood of $\bar{\Delta}_{2}$ (we recall that $\Omega_{0}$ was defined as $\mathbb{C}^{2}$ with coordinate functions $\left.\left(z_{1}^{(0)}, z_{2}^{(0)}\right)\right)$. This proper transform is considered after all the blow-ups we made, i.e. first at the points $\left\{a_{j}\right\}_{j \in \mathbb{Z}}$ and then $\left\{\alpha_{j}\right\}_{j \in \mathbb{Z}}$.

Let $c_{1}, \ldots, c_{n-1}$ be integers defined recursively by $c_{1}=1$ and, for $k \geq 2$, $c_{k}=2 k-1+(k-1) c_{1}+(k-2) c_{2}+\cdots c_{k-1}$. We also consider $d_{1}, \ldots, d_{n-1}$ positive integers defined by $d_{n-1}=1$ and, for $k \leq n-2, d_{k}=d_{k+1}+2 d_{k+2}+$ $\cdots(n-k-1) d_{n-1}+n-k$. Let $N=2 n\left(d_{1}+d_{2}+\cdots+d_{n-1}+1\right)$.

We define $f_{1}$ and $f_{2}$ as

$$
\begin{gathered}
f_{1}(\lambda)=\varepsilon P_{1}(\lambda) P_{2}^{2}(\lambda) \cdots P_{n-1}^{n-1}(\lambda) \cdot \lambda^{n}, \\
f_{2}(\lambda)=\varepsilon^{2} P_{1}(\lambda) P_{2}(\lambda) \cdots P_{n-1}(\lambda) \cdot \lambda
\end{gathered}
$$

where $\varepsilon$ is a positive real number that satisfies $\varepsilon<\left(\frac{1}{6}\right)^{N} \frac{1}{n+2} r$ and $P_{1}, \ldots, P_{n-1}$ are polynomials defined recursively by $P_{n-1}(\lambda)=\varepsilon^{c_{n-1}}-\lambda$ and, for $k \leq n-2$, $P_{k}(\lambda)=\varepsilon^{c_{k}}-P_{k+1}(\lambda) \cdot P_{k+2}^{2}(\lambda) \cdots P_{n-1}^{n-k-1}(\lambda) \cdot \lambda^{n-k}$

Remarks: 1) $P_{k}(0) \neq 0$ and $P_{j}$ and $P_{k}$ have no common zero for $j \neq k$.

2) Each $P_{k}$ is a monic polynomial of degree $d_{k}$.

There are four conditions that we want the sequence $\left\{g_{n}\right\}$ to satisfy:

I) $g_{n}\left(\bar{\Delta}_{2}\right) \subset \tilde{W}$. We will prove in fact that $g_{n}\left(\bar{\Delta}_{2}\right) \subset \tilde{W}_{r}^{\rho}$.

II) $\bigcup_{n>1} g_{n}\left(\Delta_{2} \backslash \Delta\right)$ is relatively compact in $\tilde{W}$

III) $g_{n \mid S^{1}}$ is uniformly convergent

IV) $\bigcup_{n \geq 1} g_{n}(\bar{\Delta})$ is not relatively compact in $\tilde{W}$.

- Because $P_{k}(0) \neq 0$, the definition of $f_{1}$ and $f_{2}$ implies that the origin $0 \in \mathbb{C}$ is a zero of order 1 for $f_{2}$ and a zero of order $n$ for $f_{1}$. This implies that $g_{n}(0) \in L_{n-1}$ and this shows that $\left\{g_{n}(0)\right\}_{n \geq 1}$ is not relatively compact in $X$. Hence $\left\{g_{n}\right\}$ satisfies property IV).

- We will prove next that $\left\{g_{n}\right\}$ satisfies properties II) and III).

Let $K_{n}:=\left\{\left(z_{1}, z_{2},\left[\xi_{1}: \xi_{2}\right] \in \Omega_{1}:\left|z_{1}\right| \leq \frac{1}{n},\left|z_{2}\right| \leq \frac{1}{n},\left|\xi_{2}\right| \leq \frac{1}{n}\left|\xi_{1}\right|\right\}\right.$. Note that $K_{n}$ is a compact subset of $X, K_{n} \supset K_{n+1}$, and $\cap_{n \geq 1} K_{n}=\{(0,0,[1: 0])\}$. Hence for $n$ large enough $K_{n} \subset \tilde{W}$. Therefore if we show that $g_{n}(\{\lambda \in \mathbb{C}$ : $1 \leq|\lambda| \leq 2\}) \subset K_{n}$ then we will prove both I) and II). 
Lemma 1. For $k \in\{1, \ldots, n-1\}$, if $P_{k}(\lambda)=0$ then $|\lambda|<\frac{1}{2^{k}}$.

Proof. We will prove our assertion by backward induction on $k$. For $k=n-1$ the statement is obvious. We assume that we have proved our assertion for $j \geq k+1$ and we prove it for $k$. For $j \geq k+1$ as $P_{j}$ are monic polynomials and all they zeros are inside the disk $\left\{|\lambda \in \mathbb{C}:| \lambda \mid<\frac{1}{2^{j}}\right\} \subset\{\lambda \in \mathbb{C}:|\lambda|<$ $\left.\frac{1}{2^{k+1}}\right\}$ we have that, for every $\lambda \in \mathbb{C}$ with $|\lambda|=\frac{1}{2^{k}},\left|P_{j}(\lambda)\right| \geq\left(\frac{1}{2}\right)^{d_{j}(k+1)}$ (see for example the proof of the next Corollary). It follows that $\mid P_{k+1}(\lambda)$. $P_{k+2}^{2}(\lambda) \cdots P_{n-1}^{n-k-1}(\lambda) \cdot \lambda^{n-k} \mid \geq \frac{1}{2^{N}}>\varepsilon>\varepsilon^{c_{k}}$ for $|\lambda|=\frac{1}{2^{k}}$. Rouché's theorem (see e.g. [8] page 106) implies that $P_{k}(\lambda)$ and $P_{k+1}(\lambda) \cdot P_{k+2}^{2}(\lambda) \cdots P_{n-1}^{n-k-1}(\lambda)$. $\lambda^{n-k}$ have the same number of zeros inside the disk $\left\{\lambda \in \mathbb{C}:|\lambda|<\frac{1}{2^{k}}\right\}$. As the two polynomials have the same degree and all the zeros of the second one are in this disk, it follows that all the zeros of $P_{k}$ are in there as well.

Corollary 1. If $\lambda \in \mathbb{C}$ satisfies $1 \leq|\lambda| \leq 2$ then $\left(\frac{1}{2}\right)^{d_{k}}<\left|P_{k}(\lambda)\right|<3^{d_{k}}$

Proof. Because $P_{k}$ is a monic polynomial of degree $d_{k}$ we have that it is of the form $P_{k}(\lambda)=\left(\lambda-\lambda_{1}^{(k)}\right) \cdots\left(\lambda-\lambda_{d_{k}}^{(k)}\right)$ where $\lambda_{j}^{(k)}$ are its roots (counted with multiplicity). Lemma 1 implies that $\left|\lambda_{j}^{(k)}\right|<\frac{1}{2^{k}} \leq \frac{1}{2}$ and therefore for $1 \leq|\lambda| \leq 2$ we have that $\frac{1}{2}<\left|\lambda_{j}^{(k)}-\lambda\right|<2+\frac{1}{2}<3$.

Given our choice of $\varepsilon$ and Corollary 1, a simple computation shows:

Corollary 2. If $\lambda \in \mathbb{C}$ satisfies $1 \leq|\lambda| \leq 2$ then we have:

a) $\left|f_{1}(\lambda)\right|<\frac{1}{n} r \leq \frac{1}{n}$

b) $\left|f_{2}(\lambda)\right|<\frac{1}{n} r^{2} \leq \frac{1}{n}$

c) $\left|f_{2}(\lambda)\right|<\frac{1}{n}\left|f_{1}(\lambda)\right|$,

d) $\left|f_{1}(\lambda)\right|>\left|f_{2}(\lambda)\right|^{k}$ for every $k \geq 1$.

As $f_{1}$ and $f_{2}$ have no zero inside $\{\lambda \in \mathbb{C}: 1 \leq|\lambda| \leq 2\}$ this last Corollary implies that $g_{n}(\{\lambda \in \mathbb{C}: 1 \leq|\lambda| \leq 2\}) \subset K_{n}$.

- We move now to the proof of property I).

Let $\mathcal{Z}=\{0\} \cup\left\{\lambda \in \mathbb{C}: \exists k\right.$ such that $\left.P_{k}(\lambda)=0\right\}$ and we make the obvious remark that $f_{1}(\lambda)=0$ if and only if $f_{2}(\lambda)=0$ if and only if $\lambda \in \mathcal{Z}$.

We we will show first that $\left(f_{1}, f_{2}\right)\left(\bar{\Delta}_{2} \backslash \mathcal{Z}\right) \subset \bigcup_{k>0} U_{r}^{(k)} \backslash L \subset \mathcal{U} \backslash L$.

We have seen that $U_{r}^{(k)} \backslash L=\left\{\left(z_{1}^{(0)}, z_{2}^{(0)}\right) \in \mathbb{C}^{2}:\left|z_{1}^{(0)}\right|<r\left|z_{2}^{(0)}\right|^{k},\left|z_{2}^{(0)}\right|^{k+2}<\right.$ $\left.r\left|z_{1}^{(0)}\right|\right\}$. We prove that $\bigcup_{k \geq 0} U_{r}^{(k)} \backslash L \supset\left\{\left(z_{1}^{(0)}, z_{2}^{(0)}\right) \in \mathbb{C}^{2}:\left|z_{1}^{(0)}\right|<r,\left|z_{2}^{(0)}\right|<\right.$ $\left.r^{2}\right\} \backslash\left\{\left(z_{1}^{(0)}, z_{2}^{(0)}\right) \in \mathbb{C}^{2}: z_{1}^{(0)}=0\right\}$. This inclusion together with Corollary 2 
implies that indeed $\left(f_{1}, f_{2}\right)\left(\bar{\Delta}_{2} \backslash \mathcal{Z}\right) \subset \mathcal{U} \backslash L$. Let $z_{1}^{(0)}, z_{2}^{(0)} \in \mathbb{C}$ be such that $0<\left|z_{1}^{(0)}\right|<r$ and $\left|z_{2}^{(0)}\right|<r^{2}$. If $z_{2}^{(0)}=0$ then obviously $\left(z_{1}^{(0)}, z_{2}^{(0)}\right) \in U_{r}^{(0)} \backslash L$. Otherwise we notice that $\frac{\left|z_{2}^{(0)}\right|^{k+2}}{r}<r\left|z_{2}^{(0)}\right|^{k}$ (we have assumed that $r<1$ ) and we let $I_{k}:=\left(\frac{\left|z_{2}^{(0)}\right|^{k+2}}{r}, r\left|z_{2}^{(0)}\right|^{k}\right) \subset \mathbb{R}$. As $\frac{\left|z_{2}^{(0)}\right|^{k+2}}{r}<r\left|z_{2}^{(0)}\right|^{k+1}$ it follows that $I_{k} \cap I_{k+1} \neq \emptyset$. At the same time $I_{0}=\left(\frac{\left|z_{2}^{(0)}\right|^{2}}{r}, r\right)$ and $\lim _{k \rightarrow \infty} \frac{\left|z_{2}^{(0)}\right|^{k+2}}{r}=0$. This implies that $\bigcup_{k \geq 0} I_{k}=(0, r)$ and therefore $\left|z_{1}^{(0)}\right| \in \bigcup_{k \geq 0} I_{k}$.

Moreover we claim that $\left(f_{1}, f_{2}\right)\left(\bar{\Delta}_{2} \backslash \mathcal{Z}\right) \subset \bigcup_{k=0}^{n-1} U_{r}^{(k)} \backslash L$. To prove this it is enough to show that for $k \geq n$ one has $\left|f_{1}(\lambda)\right| \geq r\left|f_{2}(\lambda)\right|^{k}$. However from Corollary 2, d) we have that $\left|f_{1}(\lambda)\right|>\left|f_{2}(\lambda)\right|^{k}>r\left|f_{2}(\lambda)\right|^{k}$ for $1 \leq|\lambda| \leq 2$. As $\frac{f_{2}(\lambda)^{k}}{f_{1}(\lambda)}$ is a holomorphic function for $k \geq n$, the maximum modulus principle implies that the inequality is valid on $\bar{\Delta}_{2}$.

Notice now that outside $L$ the inequality $\left|z_{1}^{(k)} \xi_{1}^{(k)}\right|<\rho\left|\xi_{2}^{(k)}-\xi_{1}^{(k)}\right|$ is equivalent to $\left|z_{1}^{(k)}\right|^{2}<\rho\left|z_{2}^{(k)}-z_{1}^{(k)}\right|$. As $\left[z_{1}^{(k)}: z_{2}^{(k)}\right]=\left[z_{1}^{(0)}:\left(z_{2}^{(0)}\right)^{k+1}\right]$ the last inequality is equivalent to $\left|z_{1}^{(0)}\right|^{2}<\rho\left|\left(z_{2}^{(0)}\right)^{k+1}-z_{1}^{(0)}\right| \cdot\left|z_{2}^{(0)}\right|^{k}$. Given the description of $U_{r}^{(k)} \cap W_{r}^{\rho}$ obtained above it suffices then to show that for every $\lambda \in \bar{\Delta}_{2} \backslash \mathcal{Z}$ that satisfies $\left|f_{1}(\lambda)\right|<r\left|f_{2}(\lambda)\right|^{k}$ and $\left|f_{2}(\lambda)\right|^{k+2}<r\left|f_{1}(\lambda)\right|$ and every $k$ with $0 \leq k \leq n-1$ we have $\left|f_{1}(\lambda)\right|^{2}<\rho\left|f_{2}(\lambda)^{k+1}-f_{1}(\lambda)\right| \cdot\left|f_{2}(\lambda)\right|^{k}$. We will distinguish two cases: $k \geq 1$ and $k=0$. For $k \geq 1$ let $A_{k}=\{\lambda \in$ $\left.\Delta_{2}:\left|f_{1}(\lambda)\right|<r\left|f_{2}(\lambda)\right|^{k}\right\}$ which is an open subset of $\mathbb{C}$. Notice that due to Corollary 2 we have that $A_{k}$ is relatively compact in $\Delta_{2}$ and therefore on $\partial A_{k}$ we have that $\left|f_{1}(\lambda)\right|=r\left|f_{2}(\lambda)\right|^{k}$. Moreover, if $j \leq k-1$ and $P_{j}(\lambda)=0$, then $\lambda \notin \bar{A}_{k}$. Hence $\frac{1}{P_{j}}$ is holomorphic on a neighborhood of $\bar{A}_{k}$

Lemma 2. $P_{k}(\lambda)$ is a divisor of $\varepsilon^{2 k-1} \cdot P_{1}^{k-1}(\lambda) \cdot P_{2}^{k-2}(\lambda) \cdots P_{k-1}(\lambda)-P_{k+1}(\lambda)$. $P_{k+2}^{2}(\lambda) \cdots P_{n-1}^{n-k-1}(\lambda) \cdot \lambda^{n-k}$.

Proof. For $k=1$ we have to show that $P_{1}(\lambda)$ is a divisor of $\varepsilon-P_{2}(\lambda) \cdots P_{n-1}^{n-2}(\lambda)$. $\lambda^{n-1}$. However, by definition $c_{1}=1$ and hence $P_{1}(\lambda)=\varepsilon-P_{2}(\lambda) \cdots P_{n-1}^{n-2}(\lambda)$. $\lambda^{n-1}$ and therefore there is nothing to prove. Suppose that $k \geq 2$. Notice that for $j \leq k-1$ we have $P_{j} \equiv \varepsilon^{c_{j}}\left(\bmod P_{k}\right)$. It follows that $\varepsilon^{2 k-1} \cdot P_{1}^{k-1}$. $P_{2}^{k-2} \cdots P_{k-1}-P_{k+1} \cdot P_{k+2}^{2} \cdots P_{n-1}^{n-k-1} \cdot \lambda^{n-k} \equiv \varepsilon^{2 k-1} \cdot \varepsilon^{(k-1) c_{1}} \cdots \varepsilon^{c_{k-1}}-P_{k+1} \cdot$ $P_{k+2}^{2} \cdots P_{n-1}^{n-k-1} \cdot \lambda^{n-k}\left(\bmod P_{k}\right)$. However $2 k-1+(k-1) c_{1}+(k-2) c_{2}+$ $\cdots c_{k-1}=c_{k}$ and therefore $\varepsilon^{2 k-1} \cdot P_{1}^{k-1} \cdot P_{2}^{k-2} \cdots P_{k-1}-P_{k+1} \cdot P_{k+2}^{2} \cdots P_{n-1}^{n-k-1}$. $\lambda^{n-k} \equiv \varepsilon^{c_{k}}-P_{k+1} \cdot P_{k+2}^{2} \cdots P_{n-1}^{n-k-1} \cdot \lambda^{n-k} \equiv 0\left(\bmod P_{k}\right)$.

Lemma 3. $\left|f_{1}(\lambda)\right|^{2} \leq \frac{\rho}{2}\left|f_{2}(\lambda)^{k+1}-f_{1}(\lambda)\right| \cdot\left|f_{2}(\lambda)\right|^{k}$ for every $\lambda \in \bar{A}_{k}$ and every $k$ with $1 \leq k \leq n-1$. 
Proof. We claim that on a neighborhood of $\bar{A}_{k}$ the meromorphic function

$$
\frac{f_{1}^{2}(\lambda)}{\left(f_{2}^{k+1}(\lambda)-f_{1}(\lambda)\right) \cdot f_{2}^{k}(\lambda)}
$$

is actually holomorphic. We consider first the case $k \leq n-2$ and we notice that

$f_{2}^{k+1}(\lambda)-f_{1}(\lambda)=\varepsilon P_{1}(\lambda) \cdot P_{2}^{2}(\lambda) \cdots P_{k+1}^{k+1}(\lambda) \cdot P_{k+2}^{k+1}(\lambda) \cdots P_{n-1}^{k+1}(\lambda) \cdot \lambda^{k+1}\left(\varepsilon^{2 k+1}\right.$. $\left.P_{1}^{k}(\lambda) \cdot P_{2}^{k-1}(\lambda) \cdots P_{k}(\lambda)-P_{k+2}(\lambda) \cdot P_{k+3}^{2}(\lambda) \cdots P_{n-1}^{n-k-2}(\lambda) \cdot \lambda^{n-k-1}\right)$.

We have seen that all zeros of $P_{k+2} \cdot P_{k+3}^{2} \cdots P_{n-1}^{n-k-2} \cdot \lambda^{n-k-1}$ are inside the disk $\left\{\lambda \in \mathbb{C}:|\lambda|<\frac{1}{2}\right\} \subset \Delta_{2}$. At the same time from the definition of $\varepsilon$ and Corollary 1 it follows that on $\{\lambda \in \mathbb{C}: 1 \leq|\lambda| \leq 2\}$ we have $\left|\varepsilon^{2 k+1} \cdot P_{1}^{k} \cdot P_{2}^{k-1} \cdots P_{k}\right|<\left|P_{k+2} \cdot P_{k+3}^{2} \cdots P_{n-1}^{n-k-2} \cdot \lambda^{n-k-1}\right|$. Rouché's theorem implies that $\varepsilon^{2 k+1} \cdot P_{1}^{k} \cdot P_{2}^{k-1} \cdots P_{k}-P_{k+2} \cdot P_{k+3}^{2} \cdots P_{n-1}^{n-k-2} \cdot \lambda^{n-k-1}$ has exactly $d_{k+2}+2 d_{k+3}+\cdots+(n-k-1) d_{n-1}+n-k-1=d_{k+1}$ zeros inside $\Delta_{2}$. Then Lemma 2 implies that $\varepsilon^{2 k+1} \cdot P_{1}^{k} \cdot P_{2}^{k-1} \cdots P_{k}-P_{k+2} \cdot P_{k+3}^{2} \cdots P_{n-1}^{n-k-2} \cdot \lambda^{n-k-1}=$ $P_{k+1} Q$ where $Q$ is a polynomial which is nonvanishing on a neighborhood of $\bar{\Delta}_{2}$. We have seen that on a neighborhood of $\bar{A}_{k}$ we have that $P_{1} \cdot P_{2}^{2} \cdots P_{k-1}^{k-1}$ is nonvanishing. Hence we it remains to show that

$$
\frac{f_{1}^{2}(\lambda)}{P_{k}^{k} \cdot P_{k+1}^{k+1} \cdot P_{k+2}^{k+1} \cdots P_{n-1}^{k+1} \cdot \lambda^{k+1} \cdot P_{k+1} \cdot P_{k}^{k} \cdot P_{k+1}^{k} \cdots P_{n-1}^{k} \cdot \lambda^{k}}
$$

is holomorphic and this follows from the definition of $f_{1}$.

For $k=n-1$ Rouche's theorem implies as above that $f_{2}^{n}-f_{1}=f_{1} \cdot Q_{1}$ where $Q_{1}=\varepsilon^{2 n-1} P_{1}^{n-1} \cdot P_{2}^{n-2} \cdots P_{n-1}-1$ is nonvanishing on a neighborhood of $\bar{\Delta}_{2}$. It remains to notice that $\frac{f_{1}}{f_{2}^{n-1}}$ is holomorphic on a neighborhood of $\bar{A}_{n-1}$ and our claim is proved.

The maximum modulus principle implies that it is enough to check our inequality on $\partial A_{k}$, hence we may assume that $\left|f_{1}(\lambda)\right|=r\left|f_{2}(\lambda)\right|^{k}$. Then it suffices to show that $r^{2}\left|f_{2}(\lambda)\right|^{2 k} \leq \frac{\rho}{2}\left(r\left|f_{2}(\lambda)\right|^{k}-\left|f_{2}(\lambda)\right|^{k+1}\right) \cdot\left|f_{2}(\lambda)\right|^{k}$. Therefore it is enough to show that $r^{2} \leq \frac{\rho}{2}\left(r-\left|f_{2}(\lambda)\right|\right)$. We have seen in Corollary 1 that $\left|f_{2}(\lambda)\right| \leq r^{2}$. This means that it is enough to show that $r^{2} \leq \frac{\rho}{2}\left(r-r^{2}\right)$ and this follows from our choice of $r$.

This Lemma takes care of the case $1 \leq k \leq n-1$. It remains to deal with $k=0$. That means that we have to show that for every $\lambda \in \bar{\Delta}_{2} \backslash \mathcal{Z}$ that satisfies $\left|f_{1}(\lambda)\right|<r$ and $\left|f_{2}(\lambda)\right|^{2}<r\left|f_{1}(\lambda)\right|$ we have $\left|f_{1}(\lambda)\right|^{2}<\rho\left|f_{2}(\lambda)-f_{1}(\lambda)\right|$. This follows from the next Lemma. 
Lemma 4. For every $\lambda \in \bar{\Delta}_{2}$ we have $\left|f_{1}(\lambda)\right|^{2} \leq \frac{\rho}{2}\left|f_{2}(\lambda)-f_{1}(\lambda)\right|$

Proof. Exactly as in the proof of Lemma 3 we get that $\frac{f_{1}^{2}(\lambda)}{f_{2}(\lambda)-f_{1}(\lambda)}$ is holomorphic on a neighborhood of $\bar{\Delta}_{2}$. Hence we have to check the inequality only on $\partial \Delta_{2}$. That is, it suffices to show that $\left|f_{1}\right|^{2}+\frac{\rho}{2}\left|f_{2}\right| \leq \frac{\rho}{2}\left|f_{1}\right|$ on $\partial \Delta_{2}$. This follows from Corollary 1 (note that the two terms appearing on the left-hand side of the inequality contain $\varepsilon^{2}$ and the one on right contains $\varepsilon$ ).

Step 3. We show that the universal covering of $\tilde{W}$ (hence of $W$ ) does not satisfy the discrete disk property.

We will show first that $\tilde{W}_{r}^{\rho}$ is simply connected. As each $\tilde{W}_{r, \rho}^{(k)}$ is simply connected, it suffices to show that $\tilde{W}_{r, \rho}^{(k)} \cap \tilde{W}_{r, \rho}^{(k+1)}=U_{r}^{(k)} \cap U_{r}^{(k+1)}$ is connected for every $k \in \mathbb{Z}$. Note that for points in $U_{r}^{(k)} \cap U_{r}^{(k+1)}$ we have that $\xi_{2}^{(k)} \neq 0$, $\xi_{1}^{(k+1)} \neq 0, \xi_{1}^{(k+2)} \neq 0$. Hence $U_{r}^{(k)} \cap U_{r}^{(k+1)} \subset \mathbb{C}^{2}$ where the coordinate functions on $\mathbb{C}^{2}$ are $x:=\frac{\xi_{1}^{(k)}}{\xi_{2}^{(k)}}$ and $y=\frac{\xi_{2}^{(k+1)}}{\xi_{1}^{(k+1)}}$. In this coordinates we have the following: $z_{2}^{(k)}=z_{2}^{(k+1)}=x y, z_{1}^{(k)}=x^{2} y, z_{1}^{(k+1)}=x, \frac{\xi_{2}^{(k+2)}}{\xi_{1}^{(k+2)}}=x y^{2}$. Therefore $U_{r}^{(k)} \cap U_{r}^{(k+1)}=\left\{(x, y) \in \mathbb{C}^{2}:|y|<r,\left|x^{2} y\right|<r\right\} \cap\left\{(x, y) \in \mathbb{C}^{2}:\left|x y^{2}\right|<r,|x|<r\right\}$.

If $|x|<r$ and $|y|<r$ then $\left|x^{2} y\right|<r^{3}<r$ and $\left|x y^{2}\right|<r^{3}<r$ because we have assumed that $r<1$. it follows that

$$
U_{r}^{(k)} \cap U_{r}^{(k+1)}=\left\{(x, y) \in \mathbb{C}^{2}:|y|<r,|x|<r\right\} .
$$

In particular $U_{r}^{(k)} \cap U_{r}^{(k+1)}$ is connected (even contractible).

We proved that $g_{n}\left(\bar{\Delta}_{2}\right) \subset \tilde{W}_{r}^{\rho}$. It follows that the universal cover of $\tilde{W}$ (which contains $\tilde{W}_{r}^{\rho}$ ) does not satisfy the discrete disk property.

\section{References}

[1] M. Colţoiu: Coverings of 1-convex manifolds with 1-dimensional exceptional set. Comment. Math. Helv. 68 (1993), no. 3, 469-479.

[2] M. Colţoiu, C. Joiţa: The disk property of coverings of 1-convex surfaces. Preprint, arXiv:1104.1077v1. To appear in Proceedings of the A.M.S. 
[3] F. Docquier, H. Grauert: Levisches Problem und Rungescher Satz für Teilgebiete Steinscher Mannigfaltigkeiten. Math. Ann. 140 (1960) 94123.

[4] J. E. Fornaess: 2 dimensional counterexamples to generalizations of the Levi problem. Math. Ann. 230 (1977), no. 2, 169-173

[5] H. Grauert: Über Modifikationen und exzeptionelle analytische Mengen. Math. Ann. 146 (1962) 331-368.

[6] K. Fritzsche; H. Grauert: From holomorphic functions to complex manifolds. Graduate Texts in Mathematics, 213. Springer-Verlag, New York, 2002.

[7] T. Napier: Convexity properties of coverings of smooth projective varieties. Math. Ann. 286 (1990), no. 1-3, 433-479.

[8] R. Narasimhan: Complex analysis in one variable. Birkhäuser Boston, Inc., Boston, MA, 1985.

\section{Mihnea Colţoiu}

Institute of Mathematics of the Romanian Academy

P.O. Box 1-764, Bucharest 014700

ROMANIA

E-mail address: Mihnea.Coltoiu@imar.ro

Cezar Joiţa

Institute of Mathematics of the Romanian Academy

P.O. Box 1-764, Bucharest 014700

ROMANIA

E-mail address: Cezar.Joita@imar.ro 